\title{
Convergence of gene and cell therapy
}

\author{
Alexey Bersenev \& Bruce L Levine**
}

Gene therapy and cell therapy have followed similar roller coaster paths of rising public expectations and disappointment over the past two decades. There is now reason to believe that momentum in the field has reached the point where the successes will be more frequent. The use of gene-modified cells has opened new avenues for engineering desired cell properties, for the use of cells as vehicles for gene delivery, and for tracking cells and controlling cell persistence after transplantation. Some notable recent clinical developments in cellular engineering by gene transfer offer lessons on how the field has emerged, and hint at additional future clinical applications.

KEYWORDS: cell therapy — clinical trial • gene therapy • immunotherapy

In this article we will review some of the key developments in cell engineering by gene transfer, summarized in Box 1.

\section{Cell gene therapy for correction} of genetic disorders

The first use of gene-modified cells was for correction of an inherited missing gene function by autologous transplantation of gene-modified hematopoietic stem/progenitor cells (HSPCs). Adenosine deaminase (ADA) deficiency results in a buildup of cellular dATP and prevention of DNA synthesis. Rapidly dividing cells of the immune system and especially $\mathrm{T}$ lymphocytes are affected. As a monogenic disease, severe combined immunodeficiency (SCID)-ADA was a prime candidate for gene therapy and the first-in-human studies were performed in the early 1990s [1]. Since that time, approximately 100 transplants of gene-modified $\mathrm{CD} 4^{+}$hematopoietic cells have been performed around the world $[2,3]$. Long-term observation of patients with SCID-ADA has shown that cellular gene therapy is safe, leads to effective immunological and metabolic correction and can be an alternative to conventional HSPC transplantation $[1,4,5]$.

In the last two decades, gene correction has been clinically tested in a number of other monogenic blood/ immune disorders, such as X-linked defect in the IL-2 receptor $\gamma$ chain SCID (SCID-X1), chronic granulomatous deficiency (CGD) and Wiskott-Aldrich syndrome. Long-term results of the early trials, which utilized gammaretrovirusmediated gene therapy in autologous hematopoietic CD $34^{+}$cells in SCID-X1 patients, were published recently [6]. Transplantation of autologous genetransduced $\mathrm{CD}_{3} 4^{+}$cells led to functional correction of immune cells and resolution of clinical symptoms in patients with Wiskott-Aldrich syndrome [7]. A number of children with CGD have been treated by genemodified hematopoietic cells and showed functional recovery of myelopoiesis and resolution of infections $[8,9]$. Overall, the early clinical results of cell gene therapy of monogenic immune disorders are very promising. The majority of patients were able to recover immune function and many have been able to reduce or discontinue enzyme-replacement therapy and significantly improve their quality of life. Long-term follow-up data provides evidence for persistence of transduced T-cell lineages at least 3-5 years later and even up to 12 years after transplantation [1]. However, some cases of gamma-retrovirus-induced acute leukemia and myelodysplasia were reported in the SCID-X1 and CGD trials, respectively $[2,3,10]$. The T-cell proliferation seen in the SCID-X1 trial is likely due not only to insertional mutagenesis but also some combination of gene insertion to hematopoietic stem cells, the effects of the transgene used in this treatment, and the profound immunosuppressed status of the patients [10]. Although most of the leukemia cases have been successfully treated, it was a big setback for the field. New generations of viral vectors and other approaches to gene modification of HSPCs are now in development and clinical testing to improve safety $[2,11,12]$. These include the use of self-inactivating retroviral vectors and lentiviral vectors $[12,13]$. A study in mice comparing the tumorigenicity of

Alexey Bersenev, Department of Pathology and Laboratory Medicine, The University of Pennsylvania Perelman School of Medicine, Philadelphia, PA 19104, USA

*Author for correspondence: Bruce L Levine, Department of Pathology and Laboratory Medicine, The University of Pennsylvania Perelman School of Medicine, Philadelphia, PA 19104, USA; Tel.: +1 215573 6788; Fax: +1 215615 4718;

levinebl@mail.med.upenn.edu 
retroviral vectors to lentiviral vectors demonstrated that lentiviral vector gene transfer into hematopoietic stem cells was not tumorigenic in contrast to retroviral vectors [14]. While a degree of risk of insertional oncogenesis in HSPCs is likely to remain with currently available gene vectors, the risk of death in patients with SCID-X1 who do not have a tissue-matched donor for HSPC transplantation is unfortunately higher than the observed rate of leukemias in these early gene correction studies.

\section{Redirecting immune cell function}

One strategy to produce gene-modified cells redirected towards pathogens is the chimeric antigen receptor (CAR) approach first described by Eshhar [15] CARs express an extracellular ligand generally derived from an antibody and intracellular signaling modules derived from T-cell-signaling proteins (Figure 1 and reviewed in $[16,17])$. CARs use patientor donor-derived lymphocytes that are gene modified to express chimeric receptor genes. This combines the effector functions of T lymphocytes with the ability of antibodies to recognize surface antigens with high specificity in a non-MHC restricted manner [18]. Thus, universal targeting vectors can be constructed. The first clinical trials of CAR-based T-cell therapy for cancer were reported in 2006; by one group targeting folate receptor in ovarian cancer [19], and a separate center targeting carbonic anhydrase IX (CAIX) in metastatic renal cancer patients [20]. The CAIX is instructive in that the antigen targeted is overexpressed on renal cell carcinoma, while on normal cells expression is limited to the kidney and the biliary tract. Infusions of the CAIX CAR T cells were initially well tolerated. However, after several infusions most patients developed on-target, off-organ liver enzyme elevations and cellular and humoral immune responses against the CAR and vector [21]. The authors concluded that the liver toxicity was most likely due to the reactivity of CARs against the target

Box 1. Notable developments in the convergence of cell and gene therapy.

Correction of a faulty gene and/or missing function

- The earliest application, and perhaps to this day what the lay public associates with the term 'gene therapy'

\section{Redirecting cell function}

- Increases the number of cells that can fight disease through gene transfer to redirect and enhance function

\section{Engineering HIV resistance}

- Gene transfer of a chimeric enzyme recapitulating a naturally occurring mutation that renders homozygotes highly resistant to the most commonly transmitted tropic strain of HIV

\section{Designing cell fate: safety switches \& pathotropism}

- Enhanced function is good; uncontrolled enhanced function is not so good. Gene transfer of safety switches adds a measure of control

- A complement to redirecting target specificity is directing/redirecting cell trafficking or tropism

antigen expressed on normal biliary tract tissue. In children, results from a clinical trial using CAR's directed to the diasialoganglioside GD2 in refractory neuroblastoma have demonstrated safety of GD2-CARs [22,23].

There are a number of clinical trials testing second-generation CARs that incorporate multiple signaling domains, primarily in $\mathrm{CD}^{+} 9^{+}$and $\mathrm{CD}^{2} 0^{+}$hematologic malignancies [17]. Early clinical trials assessing CAR $\mathrm{T}$ cells against $\mathrm{B}$-cell malignancies yielded somewhat disappointing results, perhaps due to low levels of in vivo persistence. Our center is conducting a clinical trial utilizing the CAR T-cell approach targeting the CD19 antigen in B-cell leukemia and lymphoma (NCT00891215). Figure 2 shows our method for the production of CAR $\mathrm{T}$ cells. This was the first trial using lentiviral vector technology in cancer and the first to include the 4-1BB signaling domain. The engineered $\mathrm{T}$ cells expanded more than 1000fold in vivo, trafficked to bone marrow and continued to express functional CARs that have persisted at high levels. Preliminary results reported from this trial are promising as three out of three patients with advanced chronic lymphocytic leukemia treated gained clinical benefit, including complete remission in two out of the three patients $[24,25]$. These two patients have a continuing remission at $>20$ months following CAR infusion and additional patients have recently been treated. CAR T cells have the potential to last many years and provide a memory response against tumor or infection. In a series of trials testing a gp120directed CAR in HIV-positive study subjects, we recently reported decadelong persistence of CAR T cells [26]. This study also indicated the safety of gene-modified $\mathrm{T}$ cells. Insertional mutagenesis, which caused leukemia in HSPC-based trials, appears not to be a general feature of gammaretroviral vectors in this cohort with a combined total of $>500$ years of subject follow-up.

Two recent studies have reported fatal serious adverse events following CAR infusion. In a trial testing CAR T cells targeted against CD19 in B-cell lymphoma, one patient demonstrated elevated cytokines that may have been secondary to a prior subacute infection exacerbated by the immune suppression associated with chemotherapy-induced lymphodepletion. This was attributed as the most likely cause of death and 'possibly related' to CAR T-cell infusion [27]. The second case was a trial in cancer patients with overexpressing HER-2/neu tumors treated with an 


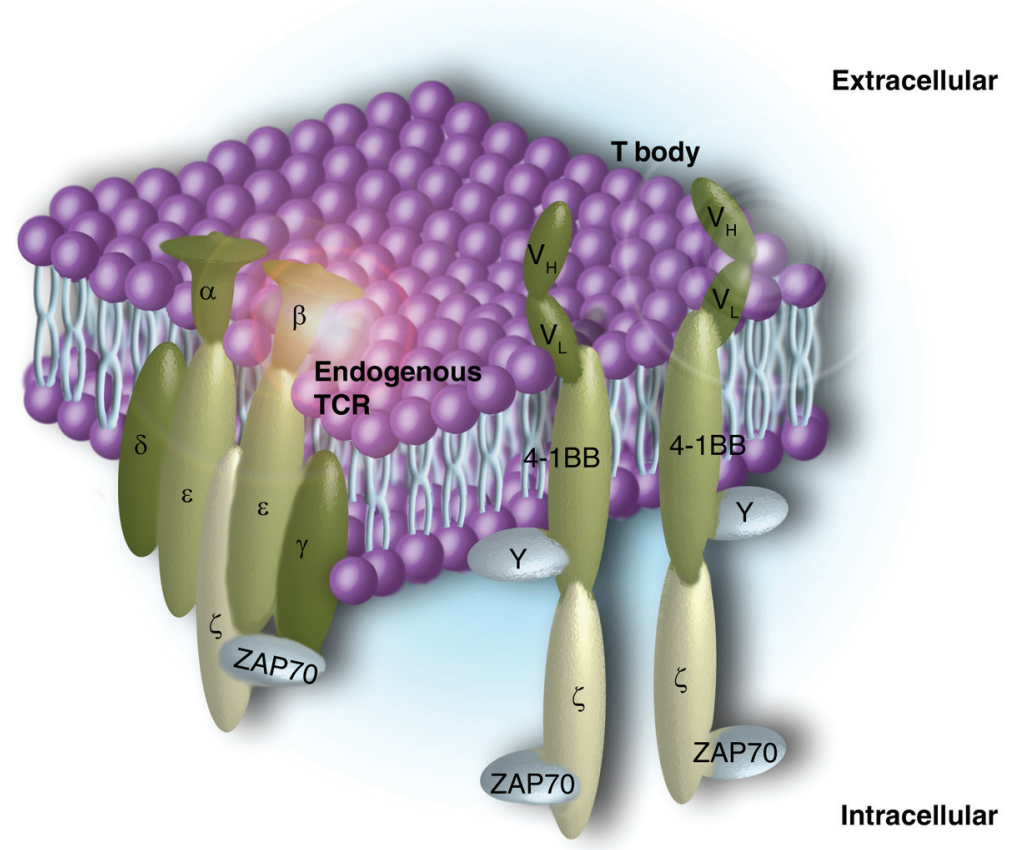

Figure 1. T cells can be engineered to have retargeted specificity for tumors. Endogenous T cells express a single heterodimeric TCR (left). Chimeric antigen receptor $T$ cells are created by the introduction of genes that encode chimeric tumor antigen-specific receptors, or T bodies, composed of antibody extracellular domains and T-cell-signaling intracellular domains such as 4-1BB and CD3 $\zeta$. Chimeric antigen receptor T cells target surface antigens in an MHC-independent fashion.

TCR: T-cell receptor; ZAP70: Zeta-chain-associated protein kinase $70 \mathrm{kDa}$.

anti-HER-2/neu CAR [28]. The patient received a lymphodepleting regimen followed by $10^{10}$ HER-2/neu CAR $\mathrm{T}$ cells. The patient demonstrated a dramatic rise in proinflammatory cytokines, consistent with a cytokine storm, initiating multisystem organ failure. In our study at the University of Pennsylvania (PA, USA), we reported delayed tumor lysis syndrome after CAR T-cell infusion [24,25], fortunately followed by recovery and remission. These cases point out the potency of this approach and the need for careful design, conduct and monitoring after infusions.

\section{Engineering HIV resistance}

Despite the profound successes of combination antiretroviral drug therapy in HIV, patients remain at risk for long-term comorbidities and uniformly exhibit recurrent viremia when antiretroviral therapy is discontinued due its failure to eradicate the viral reservoirs [29]. Cellular gene therapy approaches developed for treatment of HIV include engineering HIV resistance at various points during the HIV infection cycle, the generation of cytotoxic $\mathrm{CD}^{+}$ $\mathrm{T}$ cells expressing HIV-specific T-cell receptors, engineering HIV resistance through ribozymes, combinatorial approaches, and blockade of viral entry into immune cells by engineering HIV-resistant T cells [30].

Genetic epidemiology has led to the development of strategies to target HIV cellular entry. A naturally occurring mutation of the HIV cellular entry coreceptor CCR5
$(\Delta 32)$ was described in 1996, which explains why certain high-exposure risk patients remained HIV-resistant [31]. CCR5 was therefore recognized as a potential drug target and several drugs are now approved to block HIV cellular entry. A recapitulation of the naturally occurring mutation was reported in an HIV-positive patient in Berlin diagnosed with leukemia who underwent a HSPC treatment with a matched unrelated donor also selected for the CCR5 $\Delta 32$ mutation [32]. Several years after this treatment, there is no detectable HIV in this patient in multiple biopsied tissues [33].

Designer zinc finger nucleases (ZFNs) are chimeric DNA-binding proteins/endonucleases that can edit the genome by targeted DNA doublestrand breaks [34]. We previously showed that engineered ZFNs targeting human CCR5 efficiently generate a double-strand break at a predetermined site in the CCR5 coding region upstream of the natural CCR5 $\Delta 32$ mutation. Transient expression of the CCR5-targeted ZFNs was sufficient to selectively, efficiently and stably modify the CCR5 locus. ZFN-modified T-cells show a marked growth advantage when challenged both in vitro and in vivo with CCR5-tropic HIV [35]. CCR5 ZFN modification in CD4 T cells may render a survival advantage to these cells in HIV-infected subjects. Two clinical trials have been conducted to test the effect of these genome-modified cells on safety, increases in CD4, persistence and trafficking of CCR5 ZFN modified $T$ cells, and effect on HIV viral load (NCT01044654 and NCT00842634). The safety results have been encouraging and additional clinical trials are planned. The development of cell-based engineering approaches inducing robust $\mathrm{CD}^{+}$ T-cell resistance to HIV infection would be significantly less expensive and less toxic than allogeneic HSPC transplant with a $C C R 5^{-1-}(\triangle 32)$ donor, and less expensive than a lifetime of antiretroviral drug therapy. 


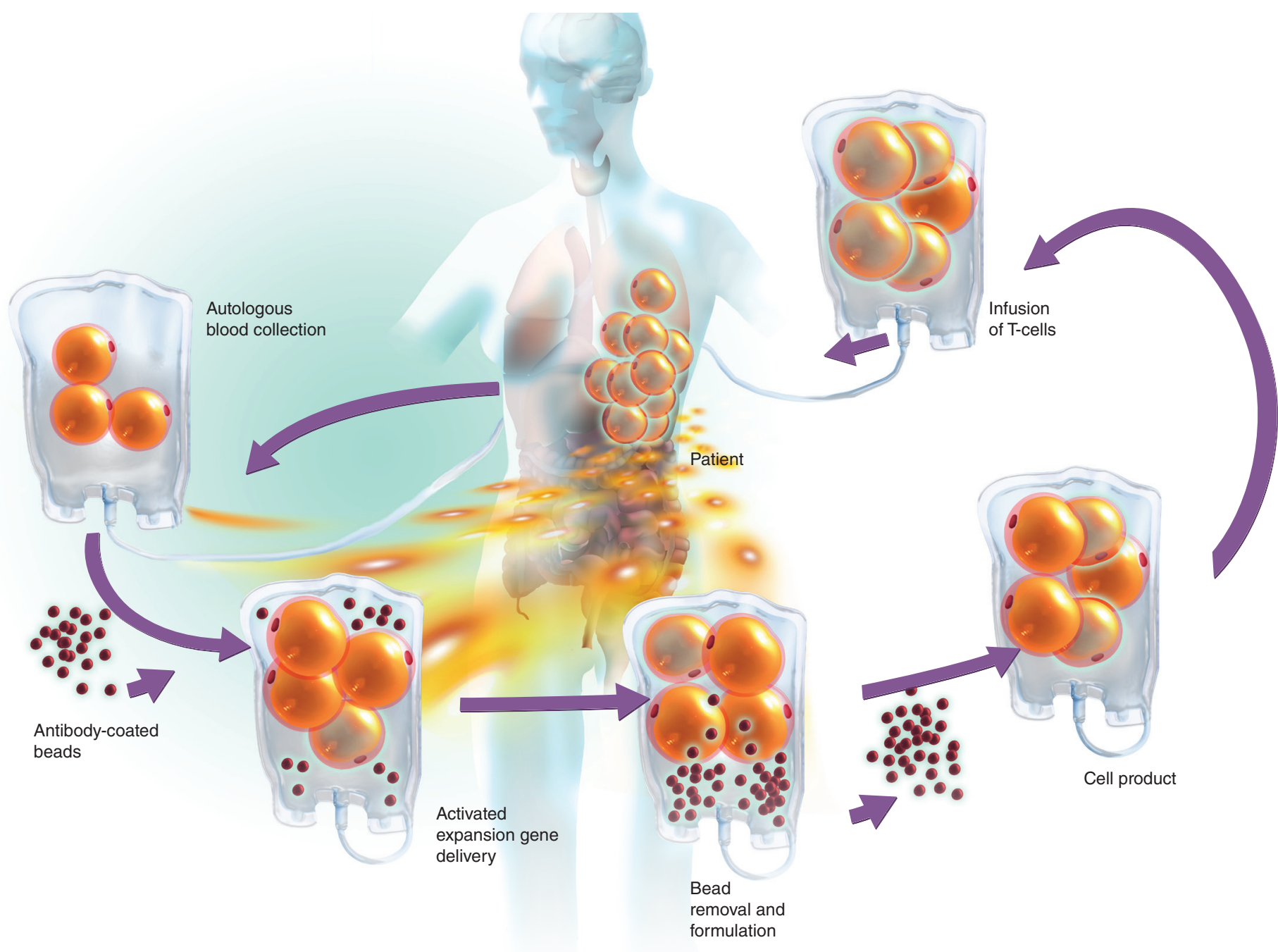

Figure 2. Ex vivo process for engineered T-lymphocyte therapies. White blood cells are removed by blood draw or leukapheresis and T cells are stimulated with anti-CD3 and anti-CD28 mAb-coated beads in media supplemented with vector encoding the transgene. Cells are expanded ex vivo for approximately 10 days when beads are removed and the cells are washed, concentrated and formulated to the final cryopreserved cell product. The cells are infused following completion and review of quality control testing.

\section{Safety switches \& pathotropism}

The ability to design and control cell fate after transfusion could prevent complications, such as inefficient migration, excessive proliferation, ontarget but off-tumor immune reactivity and graft-versus-host immune reactions. One current approach to directing cell fate involves the introduction of so-called 'suicide genes', which when activated by a drug, lead to elimination of the transduced cells. This approach has been clinically tested in donor T-cell infusions after hematopoietic stem cell transplantation in leukemia. The first clinical trials involved the gene encoding herpes simplex virus thymidine kinase as a ganciclovir-induced suicide gene [36]. The engineered $T$ cells were controlled by induction of a suicide gene in leukemic patients who had developed graft-versus-host disease (GVHD) after hematopoietic stem cell transplantation and donor lymphocyte infusion. A subsequent trial [37] showed that acute and chronic GVHD could be controlled, and a randomized open-label Phase III trial is in progress (NCT00914628). Another 'safety switch' approach has recently been studied where apoptosis has been induced in caspase- 9 genemodified $\mathrm{T}$ cells administered to patients with GVHD [38]. A single infusion of the apoptosis inducer AP1903, an otherwise bioinert smallmolecule dimerizing drug, led to rapid elimination of $90 \%$ of transduced $\mathrm{T}$ cells with resolution of GVHD. A different safety strategy is to generate chimeric antigen receptor-targeted T cells using RNA [39]. This approach avoids the use of integrating vectors and the potential for genotoxicity, reduces the costs associated with retroviral and lentiviral vector production and testing, 
and results in self-limiting expression lasting only several days in vivo. This platform provides a relatively fast, inexpensive and potentially less toxic way to test new construct designs and therapeutic targets. A clinical trial of RNA-modified T cells redirected toward mesothelin is currently underway in mesothelioma (NCT01355965).

Some cells, such as mesenchymal stromal cells and neural stem cells, have the ability to specifically migrate to an inflamed area or a tumor, described as 'pathotropism'. This ability has been exploited to selectively deliver a therapeutic gene to metastatic solid tumors in animal experiments [40,41]. Based on pathotropism, genetically engineered stem cells could be used as a vehicle to deliver a gene encoding an enzyme that can convert a nontoxic prodrug into a toxic agent and, therefore, mediate killing of the tumor mass [42]. Genetically modified neural stem cells that convert 5-fluorocytosine into the chemotherapeutic agent 5-FU are undergoing clinical trials in patients with gliomas (NCT01172964). Cell migration may be engineered in combination with any of the approaches discussed above to improve homing and engraftment and engineered cells [43].

\section{Future perspective}

From the initial idea of gene therapy as gene correction, proof-of-concept has now been demonstrated in a number of gene-modified cellular therapy approaches. The development of improved vectors and gene transfer technologies, along with improvements to ex vivo culture systems have enabled the field to blossom into a multiplicity of applications in a wide variety of diseases. What began as elegant simplicity has now evolved into the creative combination of a variety of gene and cell engineering technologies. The next step in gene and cell therapy will be to move more of these technologies into later-stage clinical trials and subsequent regulatory approval. This type of ex vivo genetic manipulation and adoptive therapy is most often autologous or directed, and is a different paradigm in drug development compared with biologics or allogeneic cell therapies. Clinical and commercial development of these technologies requires 'outscaling' and strict chain of custody practices. In the nearer term, one can speculate that the first approved genetically modified cell products will be developed for clear unmet medical needs and orphan indications, such as many of the monogenic diseases. Alternatively, products that demonstrate efficacy where repeated courses of other conventional therapies have failed will spur investment, development, regulatory approval, and commercialization. Within these diverse environments, gene and cell therapy has demonstrated the conversion of innovation into new therapies to treat our patients.

\section{Acknowledgements \\ The authors would like to thank Anne Chew for} thoughtful advice.

Financial \& competing interests disclosure

Bruce Levine has financial interest due to intellectual property and patents in the field of cell and gene therapy. Conflict of interest is managed in accordance with University of Pennsylvania policy and oversight. The authors have no other relevant affiliations or financial involvement with any organization or entity with a financial interest in or financial conflict with the subject matter or materials discussed in the manuscript apart from those disclosed.

No writing assistance was utilized in the production of this manuscript.

\section{Key points}

- Gene-modified hematopoietic cells can correct a faulty gene and/or missing function. Gene modified cells or their progeny can persist for decades.

- Immune cells can be redirected to tumor or other antigens through the delivery of genes encoding chimeric antigen receptors. Gene transfer and ex vivo expansion of the modified cells increases the number of cells that can fight disease, redirects and enhances immune cell function.

- Immune cells that are the target of HIV infection can be genetically edited to remove the most common coreceptor that HIV needs for cellular entry. This mimics a naturally occurring mutation that renders homozygotes highly resistant to the most commonly transmitted tropic strain of HIV.

- Ex vivo gene transfer of safety switches adds a measure of control to genetically modified and adoptively transferred cells. Recent work aims to control and direct cell trafficking or tropism for targeted delivery of a modified cell or prodrug.

\section{References}

Papers of special note have been highlighted as: - of interest

1 Muul LM, Tuschong LM, Soenen SL et al. Persistence and expression of the adenosine deaminase gene for 12 years and immune reaction to gene transfer components: long-term results of the first clinical gene therapy trial. Blood 101(7), 2563-2569 (2003).

Follow-up report on the first clinical trial of gene-modified $T$ cells shows the persistence of these cells for 12 years in a highly selective in vivo environment of congenital adenosine deaminase deficiency. Currently, gene-modified cells can be detected in each patient more than $\mathbf{2 0}$ years after infusion (L MUUL, PERSONAL COMMUNication).

2 Riviere I, Dunbar CE, Sadelain M. Hematopoietic stem cell engineering at a crossroads. Blood 119(5), 1107-1116 (2012). 
3 Aiuti A, Roncarolo MG. Ten years of gene therapy for primary immune deficiencies. Hematology Am. Soc. Hematol. Educ. Program 682-689 (2009).

4 Aiuti A, Cattaneo F, Galimberti S et al. Gene therapy for immunodeficiency due to adenosine deaminase deficiency. $N$. Engl. J. Med. 360(5), 447-458 (2009).

5 Gaspar HB, Cooray S, Gilmour KC et al. Hematopoietic stem cell gene therapy for adenosine deaminase-deficient severe combined immunodeficiency leads to longterm immunological recovery and metabolic correction. Sci. Transl. Med. 3(97), 97ra80 (2011).

- Reports from the Milan [3,4] and London [5] groups demonstrating effective use of adenosine deaminase (ADA) gene therapy in children with severe combined immunodeficiency (SCID)-adenosine deaminase deficiency.

6 Hacein-Bey-Abina S, Hauer J, Lim A et al. Efficacy of gene therapy for X-linked severe combined immunodeficiency. N. Engl. J. Med. 363(4), 355-364 (2010).

- Following the report of the development of leukemia in SCID-K1 children treated with gene therapy $[10]$, this report captured 10 years of follow up. Gene therapy was initially successful at correcting immune dysfunction in eight of the nine patients. However, acute leukemia developed in four patients, and one died.

7 Boztug K, Schmidt M, Schwarzer A et al. Stem-cell gene therapy for the WiskottAldrich syndrome. N. Engl. J. Med. 363(20), 1918-1927 (2010).

- Children with Wiskott-Aldrich syndrome treated with gene therapy saw their clinical condition markedly improve.

8 Kang EM, Choi U, Theobald N. Retrovirus gene therapy for X-linked chronic granulomatous disease can achieve stable long-term correction of oxidase activity in peripheral blood neutrophils. Blood 115(4), 783-791 (2010).

9 Ott MG, Schmidt M, Schwarzwaelder K et al. Correction of X-linked chronic granulomatous disease by gene therapy, augmented by insertional activation of MDS1-EVI1, PRDM16 or SETBP1. Nat. Med. 12(4), 401-409 (2006).

10 Hacein-Bey-Abina S, von Kalle C, Schmidt $\mathrm{M}$ et al. LMO2-associated clonal $\mathrm{T}$ cell proliferation in two patients after gene therapy for SCID-X1. Science 302(5644), 415-419 (2003).

- Following the development of leukemia in patients with severe combined immunodeficiency that were administered retrovirally transduced hematopoietic stem cells, clonal proliferation of $\mathrm{T}$ cells was detected. Tracking T-cell clonality and integration site analysis is an important part of longterm follow-up in gene transfer studies.

11 Kay MA. State-of-the-art gene-based therapies: the road ahead. Nat. Rev. Genet. 12(5), 316-328 (2011).

12 Scaramuzza S, Biasco L, Ripamonti A et al. Preclinical safety and efficacy of human CD34(+) cells transduced with lentiviral vector for the treatment of Wiskott-Aldrich syndrome. Mol. Ther. doi:10.1038/ mt.2012.2310 (2012) (Epub ahead of print).

13 Booth C, Gaspar HB, Thrasher AJ. Gene therapy for primary immunodeficiency. Curr. Opin. Pediatr. 23(6), 659-666 (2011).

14 Montini E, Cesana D, Schmidt M et al. Hematopoietic stem cell gene transfer in a tumor-prone mouse model uncovers low genotoxicity of lentiviral vector integration. Nat. Biotechnol. 24(6), 687-696 (2006).

15 Gross G, Waks T, Eshhar Z. Expression of immunoglobulin-T-cell receptor chimeric molecules as functional receptors with antibody-type specificity. Proc. Natl Acad. Sci. USA 86(24), 10024-10028 (1989).

16 Sadelain M, Brentjens R, Riviere I. The promise and potential pitfalls of chimeric antigen receptors. Curr. Opin. Immunol. 21(2), 215-223 (2009).

17 Kohn DB, Dotti G, Brentjens R et al. CARs on track in the clinic. Mol. Ther. 19(3), 432-438 (2011).

18 Pinthus JH, Waks T, Kaufman-Francis K et al. Immuno-gene therapy of established prostate tumors using chimeric receptorredirected human lymphocytes. Cancer Res. 63(10), 2470-2476 (2003).

19 Kershaw MH, Westwood JA, Parker LL et al. 2006. A Phase I study on adoptive immunotherapy using gene-modified T cells for ovarian cancer. Clin. Cancer Res. 12(20 Pt 1), 6106-6115.

20 Lamers CH, Sleijfer S, Vulto AG et al. Treatment of metastatic renal cell carcinoma with autologous T-lymphocytes genetically retargeted against carbonic anhydrase IX: first clinical experience. J. Clin. Oncol. 24(13), e20-e22 (2006).

21 Lamers CH, Willemsen R, van Elzakker P et al. Immune responses to transgene and retroviral vector in patients treated with ex vivo-engineered T cells. Blood 117(1), 72-82 (2011).

- Initial report [20] and excellent follow-up [21] studies of gene-modified T cells redirected to carbonic anhydrase IX. Following infusions, elevations in liver enzymes indicated on-target but offtumor toxicity. Humoral and/or cellular anticarbonic anhydrase IX-chimeric antigen receptor $\mathrm{T}$-cell immune responses were also observed. Two studies report on important studies following adverse clinical events $[10,21]$.

22 Pule MA, Savoldo B, Myers GD et al. Virus-specific T cells engineered to coexpress tumor-specific receptors: persistence and antitumor activity in individuals with neuroblastoma. Nat. Med. 14(11), 1264-1270 (2008).

23 Louis CU, Savoldo B, Dotti G et al. Antitumor activity and long-term fate of chimeric antigen receptor-positive $T$ cells in patients with neuroblastoma. Blood 118(23), 6050-6056 (2011).

24 Kalos M, Levine BL, Porter DL et al. $\mathrm{T}$ cells with chimeric antigen receptors have potent antitumor effects and can establish memory in patients with advanced leukemia. Sci. Transl. Med. 3(95), $95 \mathrm{ra} 73$ (2011).

- Report on first three patients treated on a clinical trial using autologous $T$ cells genetically modified with a chimeric antigen receptor (CAR) targeting a CD19 molecule on chronic lymphocytic leukemia cells. The data shows that a patient's modified T cells can survive for many months after administration, have the ability to grow in the body in large quantities, and have been able to kill large quantities of chronic lymphocytic leukemia cells in the patients.

25 Porter DL, Levine BL, Kalos M, Bagg A, $\mathrm{CH}$ June. Chimeric antigen receptormodified $\mathrm{T}$ cells in chronic lymphoid leukemia. N. Engl. J. Med. 365(8), 725-733 (2011).

26 Scholler N, Fu N, Yang Y et al. Soluble member(s) of the mesothelin/ megakaryocyte potentiating factor family are detectable in sera from patients with ovarian carcinoma. Proc. Natl Acad. Sci. USA 96(20), 11531-11536 (1999). 
27 Brentjens R, Yeh T, Bernal Y, Riviere I, Sadelain M. Treatment of chronic lymphocytic leukemia with genetically targeted autologous $\mathrm{T}$ cells: case report of an unforeseen adverse event in a Phase I clinical trial. Mol. Ther. 18(4), 666-668 (2010).

28 Morgan RA, Yang JC, Kitano M, Dudley ME, Laurencot CM, Rosenberg SA. Case report of a serious adverse event following the administration of $\mathrm{T}$ cells transduced with a chimeric antigen receptor recognizing ERBB2. Mol. Ther. 18(4), 843-851 (2010).

29 Siliciano JD, Kajdas J, Finzi D et al. Longterm follow-up studies confirm the stability of the latent reservoir for HIV-1 in resting CD4(+) T cells. Nat. Med. 9(6), 727-728 (2003).

30 Rossi JJ, June CH, Kohn DB. Genetic therapies against HIV. Nat. Biotechnol. 25(12), 1444-1454 (2007).

31 Liu R, Paxton WA, Choe S et al. Homozygous defect in HIV-1 coreceptor accounts for resistance of some multiplyexposed individuals to HIV-1 infection. Cell 86(3), 367-377 (1996).

32 Hutter G, Nowak D, Mossner M et al. Long-term control of HIV by CCR5 Delta32/Delta32 stem-cell transplantation. N. Engl. J. Med. 360 (7), 692-698 (2009).
33 Allers K, Hutter G, Hofmann J et al. Evidence for the cure of HIV infection by CCR5Delta32/Delta32 stem cell transplantation. Blood 117(10), 2791-2799 (2011).

34 Urnov FD, Miller JC, Lee YL et al. Highly efficient endogenous human gene correction using designed zinc-finger nucleases. Nature 435(7042), 646-651 (2005).

35 Perez EE, Wang J, Miller JC et al. Establishment of HIV-1 resistance in $\mathrm{CD}^{+}$ $\mathrm{T}$ cells by genome editing using zinc-finger nucleases. Nat. Biotechnol. 26(7), 808-816 (2008).

36 Tiberghien P, Ferrand C, Lioure B et al. Administration of herpes simplexthymidine kinase-expressing donor $\mathrm{T}$ cells with a T-cell-depleted allogeneic marrow graft. Blood 97(1), 63-72 (2001).

37 Ciceri F, Bonini C, Stanghellini MT et al. Infusion of suicide-gene-engineered donor lymphocytes after family haploidentical haemopoietic stem-cell transplantation for leukaemia (the TK007 trial): a nonrandomised Phase I-II study. Lancet Oncol. 10(5), 489-500 (2009).

38 Di Stasi A, Tey SK, Dotti G et al. Inducible apoptosis as a safety switch for adoptive cell therapy. N. Engl. J. Med. 365(18), 1673-1683 (2011).
39 Zhao Y, Moon E, Carpenito C et al. Multiple injections of electroporated autologous $\mathrm{T}$ cells expressing a chimeric antigen receptor mediate regression of human disseminated tumor. Cancer Res. 70 (22), 9053-9061 (2010).

40 Yin J, Kim JK, Moon JH et al. hMSCmediated concurrent delivery of endostatin and carboxylesterase to mouse xenografts suppresses glioma initiation and recurrence. Mol. Ther. 19(6), 1161-1169 (2011).

41 Joo KM, Park IH, Shin JY et al. Human neural stem cells can target and deliver therapeutic genes to breast cancer brain metastases. Mol. Ther. 17(3), 570-575 (2009).

42 Kim SU, Jeung EB, Kim YB, Cho MH, Choi KC. Potential tumor-tropic effect of genetically engineered stem cells expressing suicide enzymes to selectively target invasive cancer in animal models. Anticancer Res. 31(4), 1249-1258 (2011).

43 Sarkar D, Spencer JA, Phillips JA et al. Engineered cell homing. Blood 118(25) e184-e191 (2011). 